\title{
Association between Traffic Related Air Pollution and the Development of Asthma Phenotypes in Children: A Systematic Review
}

\author{
Nelson Lau $\mathbb{D}^{1},{ }^{1}$ Alex Norman, ${ }^{1}$ Mary Jane Smith, ${ }^{2}$ Atanu Sarkar, ${ }^{3}$ and Zhiwei Gao $\mathbb{D}^{1}$ \\ ${ }^{1}$ Department of Clinical Epidemiology, Faculty of Medicine, Memorial University of Newfoundland, \\ Newfoundland and Labrador, Canada \\ ${ }^{2}$ Discipline of Pediatrics, Faculty of Medicine, Memorial University of Newfoundland, Newfoundland and Labrador, Canada \\ ${ }^{3}$ Division of Community Health and Humanities, Faculty of Medicine, Memorial University of Newfoundland, \\ Newfoundland and Labrador, Canada
}

Correspondence should be addressed to Zhiwei Gao; zgao@mun.ca

Received 19 July 2018; Accepted 15 November 2018; Published 2 December 2018

Academic Editor: Katarzyna Zorena

Copyright (c) 2018 Nelson Lau et al. This is an open access article distributed under the Creative Commons Attribution License, which permits unrestricted use, distribution, and reproduction in any medium, provided the original work is properly cited.

Introduction. Traffic related air pollution (TRAP) has long been associated with the onset of childhood asthma. The relationship between TRAP exposure and the development of childhood asthma phenotypes is less understood. To better understand this relationship, we performed a systematic review of the literature studying childhood TRAP exposure and the development of childhood asthma and wheezing phenotypes (transient, persistent, and late-onset asthma/wheezing phenotypes). Methods. A literature search was performed in PubMed, Embase, and Scopus databases for current literature, returning 1706 unique articles. After screening and selection, 7 articles were included in the final review. Due to the low number of articles, no meta-analysis was performed. Results. TRAP exposure appears to be associated with both transient and persistent asthma/wheezing phenotypes. However, there was little evidence to suggest a relationship between TRAP exposure and late-onset asthma/wheezing. The differing results may be in part due to the heterogeneity in study methods and asthma/wheezing phenotype definitions, in addition to other factors such as genetics. Conclusion. TRAP exposure may be associated with transient and persistent asthma/wheezing phenotypes in children. The low number of studies and differing results suggest that further studies are warranted.

\section{Introduction}

Childhood asthma is the most common chronic disease in children, with estimated prevalence of $14 \%$ in children worldwide $[1,2]$. This high prevalence is also associated with significant economic burden. Asthma in school-aged children in the United States alone is estimated to cost nearly $\$ 6$ billion annually in healthcare expenditures [3]. Given the high burden of disease as well as the complex and heterogeneous nature of childhood asthma, it is essential to investigate further beyond the incidence and outcomes associated with childhood asthma [4].

Among the first studies to investigate the differences between childhood asthma symptoms was the Tucson Children's Respiratory Study, which identified 4 separate wheezing phenotypes based on the longitudinal pattern of wheezing that was observed [5]. These phenotypic groups were based on the age of wheezing onset and the duration of wheezing and included the following groups: (1) no wheezing, (2) early transient wheezing (wheezing before age of 3 but not at age of 6 years), (3) persistent wheezing (wheezing both before age of 3 and at age of 6 years), and (4) lateonset wheezing (no wheezing before age of 3 but wheezing by age of 6 years) [5]. The existence of these phenotypes has been supported by further studies, using methods such as latent class analysis and group based trajectory modelling [4, 6-8]. Currently, childhood asthma consists of many different phenotypes, each associated with differing clinical and genetic markers, risk factors, outcomes, and responses to medication $[9,10]$. Thus, understanding the different 
clinical phenotypes of childhood asthma and wheeze may lead to several benefits in diagnosis and treatment. These include knowledge of probable outcomes and prognosis, personalized treatments for patients, and understanding how environmental exposures can modify the risk of developing different childhood asthma or wheezing phenotypes [11].

Numerous studies have found traffic related air pollution (TRAP) to be associated with the onset of childhood asthma [12-16]. These results are further supported by a systematic review that showed strong associations between exposure to black carbon (BC), $\mathrm{NO}_{2}, \mathrm{PM}_{2.5}$ (atmospheric particulate matter less than $2.5 \mu \mathrm{m}$ in diameter), and $\mathrm{PM}_{10}$ (atmospheric particulate matter less than $10.0 \mu \mathrm{m}$ in diameter) with the onset of childhood asthma [17]. The association between TRAP and different childhood asthma phenotypes is less understood. Earlier reviews have focused primarily on the association between TRAP and the onset of childhood asthma rather than the development of the asthma phenotype $[17,18]$. Nevertheless, the effect of TRAP exposure on the development of different childhood asthma phenotypes may be significantly different. One study found no association between $\mathrm{NO}_{2}$ exposure and either early transient wheeze or persistent wheeze phenotypes in children [14]. These findings conflict with another study that found an association between childhood $\mathrm{NO}_{2}$ exposure and persistent wheeze in children [19]. These associations may also change with the pollutant being studied: although one study found no association between childhood $\mathrm{NO}_{2}$ exposure and early transient wheezing, it found an association between childhood $\mathrm{PM}_{2.5}$ exposure and early transient wheezing [14].

The purpose of this systematic review is to synthesize the results of observational epidemiological studies studying the association between TRAP exposure and the development of childhood asthma/wheezing phenotypes, namely, transient asthma/wheezing, late-onset asthma/wheezing, and persistent asthma/wheezing in children aged 0-18 years.

\section{Methods}

2.1. Selection Criteria. This systematic review was conducted in accordance with the Preferred Reporting Items for Systematic Reviews and Meta Analyses (PRISMA) statement for reporting systematic reviews and meta-analyses [24]. The review included cross-sectional, case-control, and cohort studies which studied the association between TRAP exposure and the development of childhood asthma phenotypes, namely, early-transient asthma, late-onset asthma, and persistent asthma in children aged 0-18 years.

Studies were included if they

(1) were epidemiological or observational studies such as cross-sectional, cohort, or case-control studies;

(2) had some measure of TRAP $\left(\mathrm{CO}, \mathrm{PM}_{2.5}, \mathrm{PM}_{10}\right.$, and $\mathrm{NO}_{2}$ ) exposure [25] for children within the early life period between fetal stage and age of 12 (through either modelling or direct measurement);

(3) examined the association between TRAP exposure and development of asthma or wheeze outcomes when the child is aged $0-18$ years;
(4) explicitly included at least one type of asthma/ wheezing phenotype (late-onset asthma/wheeze, persistent asthma/wheeze, and transient asthma/wheeze) in their outcomes.

Studies were excluded if they

(1) measured TRAP exposure only when children were aged > 12 years;

(2) were reviews, commentaries, experimental studies, letters to the editor, and so forth;

(3) were studies that only examine the association between TRAP exposure and asthma development without specifying the phenotype or look at exacerbation of asthma/wheeze, allergies, and so forth as the outcome;

(4) measured exclusively pollution exposure to nonTRAP pollutants, such as $\mathrm{O} 3$ or $\mathrm{SO} 2$;

(5) were non-English-language studies.

No studies were excluded on the basis of publication year.

2.2. Health Outcomes. The primary health outcomes assessed were childhood asthma/wheezing phenotypes. Articles with either wheezing phenotypes or asthma phenotypes as outcomes were included for analysis. Although wheezing is a nonspecific symptom that is not always associated with childhood asthma, wheezing phenotypes have long been used to characterize the corresponding childhood asthma phenotypes [18, 26, 27]. To account for the differing followup times between studies, asthma/wheezing phenotypes were divided into 3 groups with the following modified definitions based on the Tucson Children's Respiratory Study [5]:

(1) Transient asthma/wheezing: onset of asthma or wheezing before or at age of 3 and no asthma or wheezing after age 3

(2) Persistent asthma/wheezing: onset of asthma or wheezing before or at age of 3 , with evidence of asthma or wheezing after age of 3

(3) Late-onset asthma/wheezing: onset of asthma or wheezing after age of 3

2.3. Search Strategy. Searches were performed in the PubMed, Embase, and Scopus databases for relevant articles. Search strings containing terms for "asthma," "vehicle emissions," and "children" were used. An example search string for PubMed is given below:

("Asthma”[Mesh] OR asthma OR wheeze) AND ("Motor Vehicles"[Mesh] OR "Vehicle Emissions"[Mesh] OR traffic OR car OR truck OR bus OR motorcycle OR automobile OR vehicle OR exhaust) AND ("Child"[Mesh] OR "Infant"[Mesh] OR childhood OR children OR infant OR baby OR paediatric OR pediatric OR paediatrics OR pediatrics)

The search was performed in May 2018 and included papers published until May 2018. 

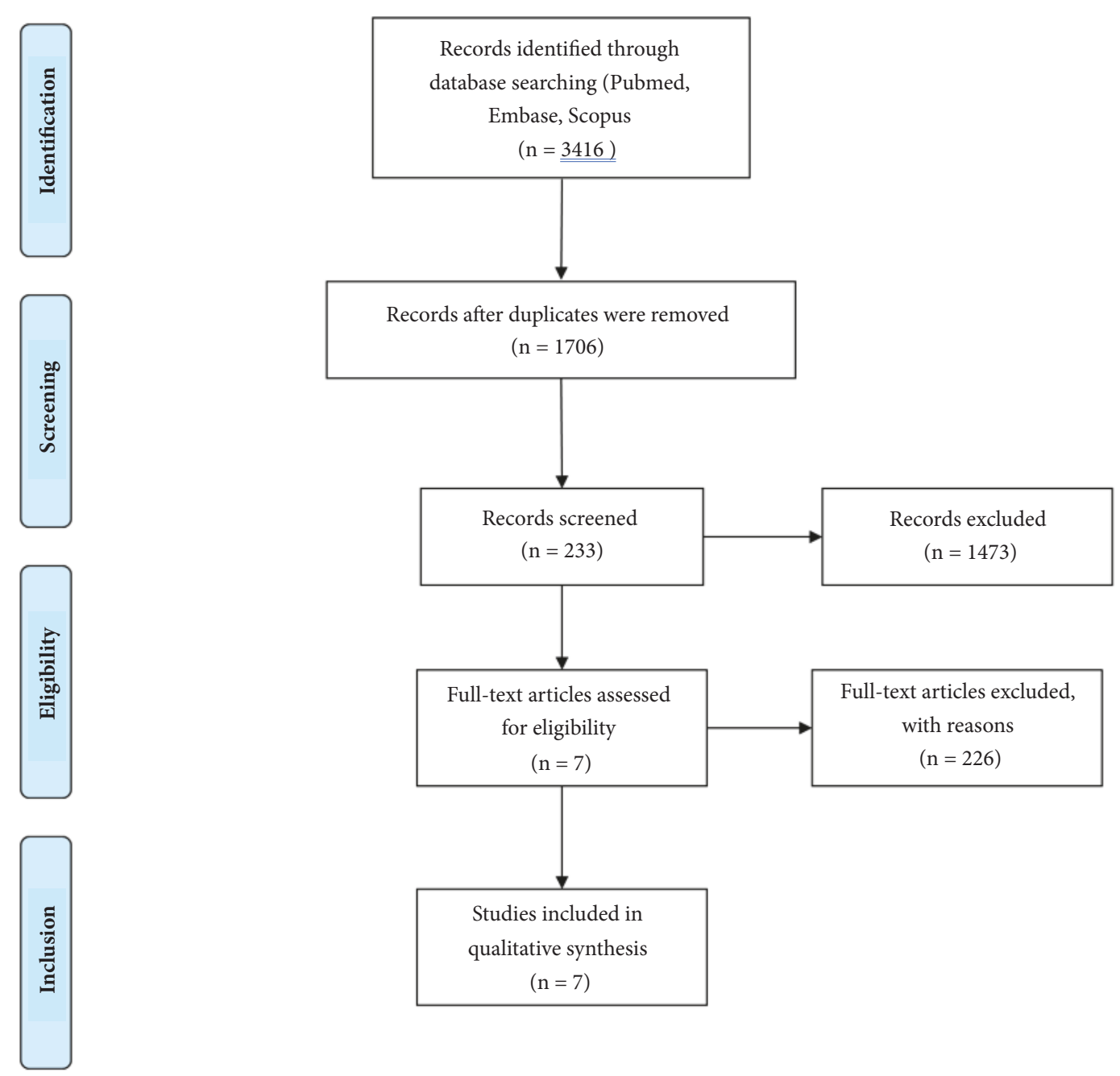

FIGURE 1: Preferred Reporting Item for Systematic Reviews and Meta-Analyses (PRISMA) flow diagram for article selection.

2.4. Quality Assessment. The Critical Appraisal Skills Programme (CASP) checklist for cohort studies was used to assess the quality of applicable studies [28]. CASP consists of 12 questions used to evaluate the quality of cohort studies. The CASP criteria were used to evaluate cohort studies for (1) selection bias in the cohorts used, (2) measurement, classification, or recall bias in exposures, (3) measurement or classification bias in outcomes, (4) adjustment for appropriate confounders, (5) length and completeness of follow-up, and (6) potential validity of results. Two reviewers (N.L. and A.N.) independently assessed each article using the CASP criteria.

2.5. Data Extraction. Relevant information was extracted independently by two reviewers (N.L. and A.N.). Information was extracted from supplementary materials when deemed necessary. Disagreements on what information to extract were resolved via consensus by both reviewers. Extracted information included authors, study location, year of publication, study design, study population, pollutant and exposure information, asthma and wheezing phenotype definitions, and outcome data.

\section{Results}

3.1. Search Results. A literature search was conducted in the PubMed, Embase, and Scopus databases, yielding 1706 unique articles. After initial screening, 233 articles were chosen for full-text review, using the selection criteria and 7 articles were deemed suitable for inclusion [4, 14, 19-23]. Figure 1 represents the PRISMA flow diagram for article selection in this study.

3.2. Study Characteristics. The 7 studies included were published from 2007 to 2018, with all being cohort studies $[4,14,19-23]$. Among the 7 studies, one birth cohort was utilized twice in separate studies [14, 19]. 111038 individuals across these 7 studies were included (duplicated cohorts were counted twice). The sample size in the included studies ranged from 2871 to 68195 individuals. Studies were conducted in Canada, France, USA, Sweden, Netherlands, and Norway and were all English language studies. The length of follow-up varied among the 7 studies, with all studies starting from birth and the end of follow-up ranging from age 4-12. 
To estimate pollutant exposure, 3 studies utilized Land Use Regression (LUR) and 4 utilized dispersion modelling. CO, $\mathrm{NO}_{2}, \mathrm{NO}_{\mathrm{x}}, \mathrm{PM}_{2.5}$, and $\mathrm{PM}_{10}$ were the traffic related air pollutants assessed. The number of studies measuring each individual pollutant is as follows:

(i) CO: 1 study

(ii) $\mathrm{NO}_{2}: 4$ studies

(iii) $\mathrm{NO}_{\mathrm{x}}: 3$ studies

(iv) $\mathrm{PM}_{2.5}: 4$ studies

(v) $\mathrm{PM}_{10}: 1$ study

Due to the differing follow-up times among the included studies, phenotypic definitions varied by study. 5 studies reported results in the form of odds ratios for asthma phenotype risk per unit of pollutant exposure $\left(\mu \mathrm{g} / \mathrm{m}^{3}\right)[14,19-$ $21,23]$. One study, that by Sbihi et al., divided the cohort into quartiles based on exposure quartiles to the lowest quartile of pollutant exposure [4]. The last study, that by Pennington et al., reported asthma phenotype risk in the form of absolute risk difference between different exposure groups [22]. Complete study characteristics, including phenotypic definitions, can be found in Table 1, while the individual results for each study can be found in Table 2 . Due to the low number of included studies, we were unable to conduct a meta-analysis.

3.3. Quality Assessment of Studies. All included studies were considered to be of sufficient quality for inclusion. The most common limitations identified from the CASP checklist were the potential for recall bias in studies where outcomes were reported via questionnaire and not adjusting for potential confounders.

\subsection{Effect of CO on Childhood Asthma Phenotype Develop-} ment. The sole study which measured CO exposure measured only persistent childhood asthma by age of 5 as a phenotypic outcome [22]. Prenatal exposure to $\mathrm{CO}$ was associated with an absolute risk increase of 3.5\% for persistent asthma at age of 5. Exposure to CO during the 1st year of life was associated with an absolute risk increase of $3.9 \%$ for persistent asthma at age of 5 .

\subsection{Effect of $\mathrm{NO}_{2}$ on Childhood Asthma Phenotype Devel-} opment. Of the four studies that measured the association between $\mathrm{NO}_{2}$ and the development of childhood asthma phenotypes, two reported associations for transient, persistent, and late-onset asthma/wheeze, one reported associations for transient and persistent wheeze, and one study reported solely the association for late-onset asthma $[4,14,19,21]$. Two studies reported results from an identical study cohort (PIAMA) $[14,19]$.

Two of the studies that listed transient asthma/wheezing as an outcome found a significant association between $\mathrm{NO}_{2}$ exposure and transient wheezing $[4,19]$, with the third study reporting no significant association [14]. However, a significant association was reported by Sbihi et al. only when the second and fourth exposure quartiles were compared to the lowest quartile. No association was observed between $\mathrm{NO}_{2}$ exposure and transient wheezing when the third exposure quartile was compared to the lowest quartile [4].

Of the three studies that reported persistent asthma/ wheezing, two studies reported no association between $\mathrm{NO}_{2}$ and persistent wheezing $[14,19]$. The third study reported significant associations for the second and third exposure quartiles compared to the reference quartile; but the highest exposure quartile was not associated with persistent asthma [4].

Two of three studies found no association between $\mathrm{NO}_{2}$ and late-onset asthma/wheezing [14, 21]. Significant associations for the second and third exposure quartiles with lateonset asthma were reported by the third study, but the highest exposure quartile was not associated with late-onset asthma [4].

3.6. Effect of $\mathrm{NO}_{x}$ on Childhood Asthma Phenotype Development. Among the three studies that measured the association between $\mathrm{NO}_{\mathrm{x}}$ exposure and the development of childhood asthma phenotypes, two studies studied transient wheezing, persistent wheezing, and late-onset wheezing as outcomes $[20,23]$. One study studied solely persistent asthma [22].

Pennington et al. found that prenatal exposure to $\mathrm{NO}_{\mathrm{x}}$ was associated with an absolute risk increase of $3.8 \%$ for persistent asthma at age of 5, while exposure to $\mathrm{NO}_{\mathrm{x}}$ during the 1st year of life was associated with an absolute risk increase of $4.0 \%$ for persistent asthma [22].

Both studies which reported odds ratios for phenotypic outcomes found a significant association between $\mathrm{NO}_{\mathrm{x}}$ exposure and persistent wheezing, with no association found with $\mathrm{NO}_{\mathrm{x}}$ and either transient or late-onset wheezing [20, 23].

3.7. Effect of $\mathrm{PM}_{2.5}$ on Childhood Asthma Phenotype Development. Among the four studies which measured the association between $\mathrm{PM}_{2.5}$ exposure and the development of childhood asthma phenotypes, two studies studied transient wheezing, persistent wheezing, and late-onset asthma or wheezing as outcomes $[4,14]$. One study reported associations for transient and persistent wheeze [19]. The final study contained solely persistent asthma as a phenotypic outcome [22]. Two studies, those by Brauer et al. and Gehring et al., reported results from an identical study cohort (PIAMA) $[14,19]$.

Pennington et al. found that prenatal exposure to $\mathrm{PM}_{2.5}$ was associated with an absolute risk increase of $4.4 \%$ for persistent asthma at age of 5 [22]. Exposure to $\mathrm{PM}_{2.5}$ during the 1st year of life was associated with an absolute risk increase of $4.5 \%$ for persistent asthma at age of 5 .

Among the three studies which reported associations between $\mathrm{PM}_{2.5}$ and transient asthma or wheezing, two reported a significant association [14, 19]. The third one by Sbihi et al. reported a significant association between transient asthma and the second and third exposure quartiles [4]. However, the highest exposure quartile was not associated with transient asthma. 


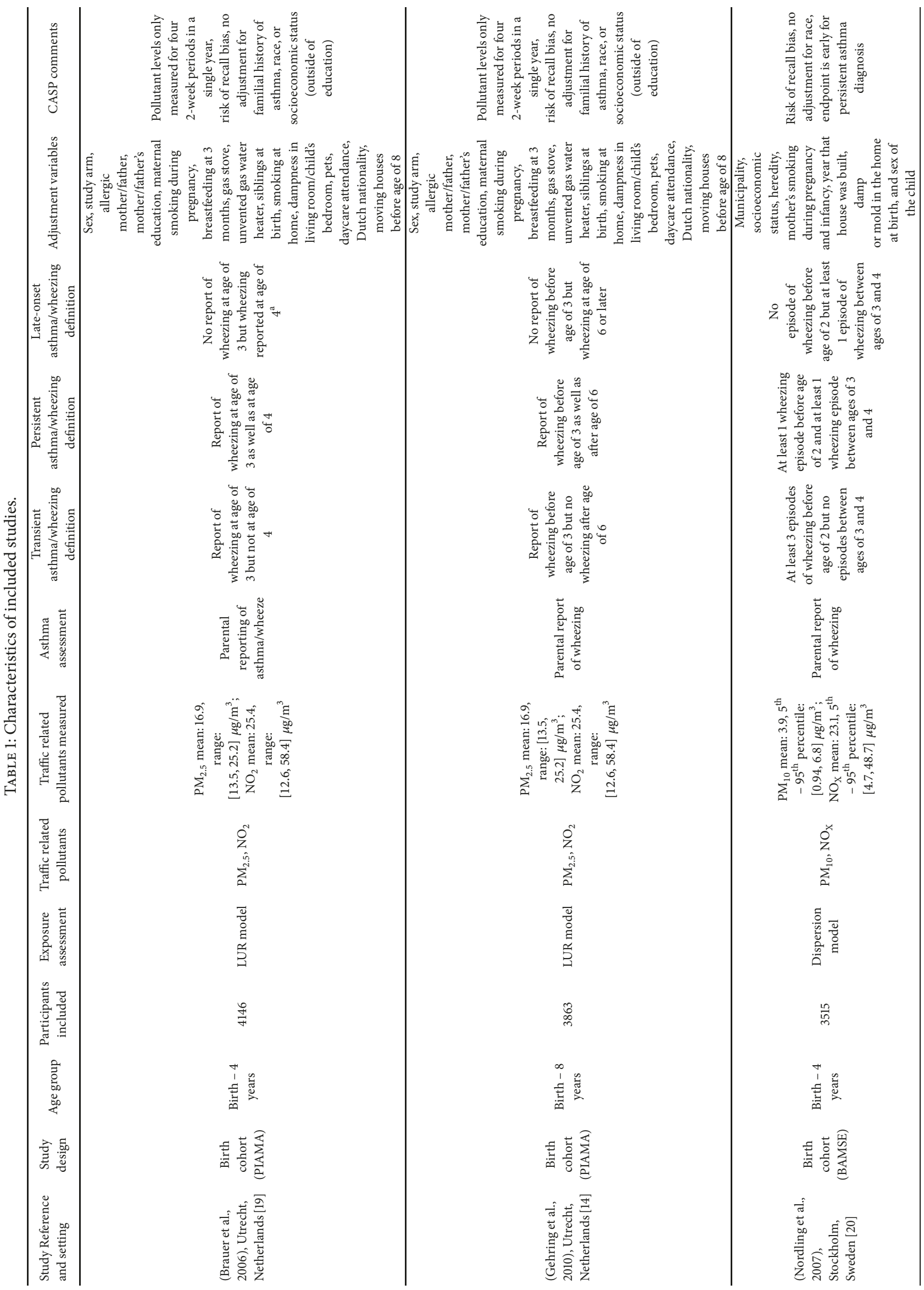




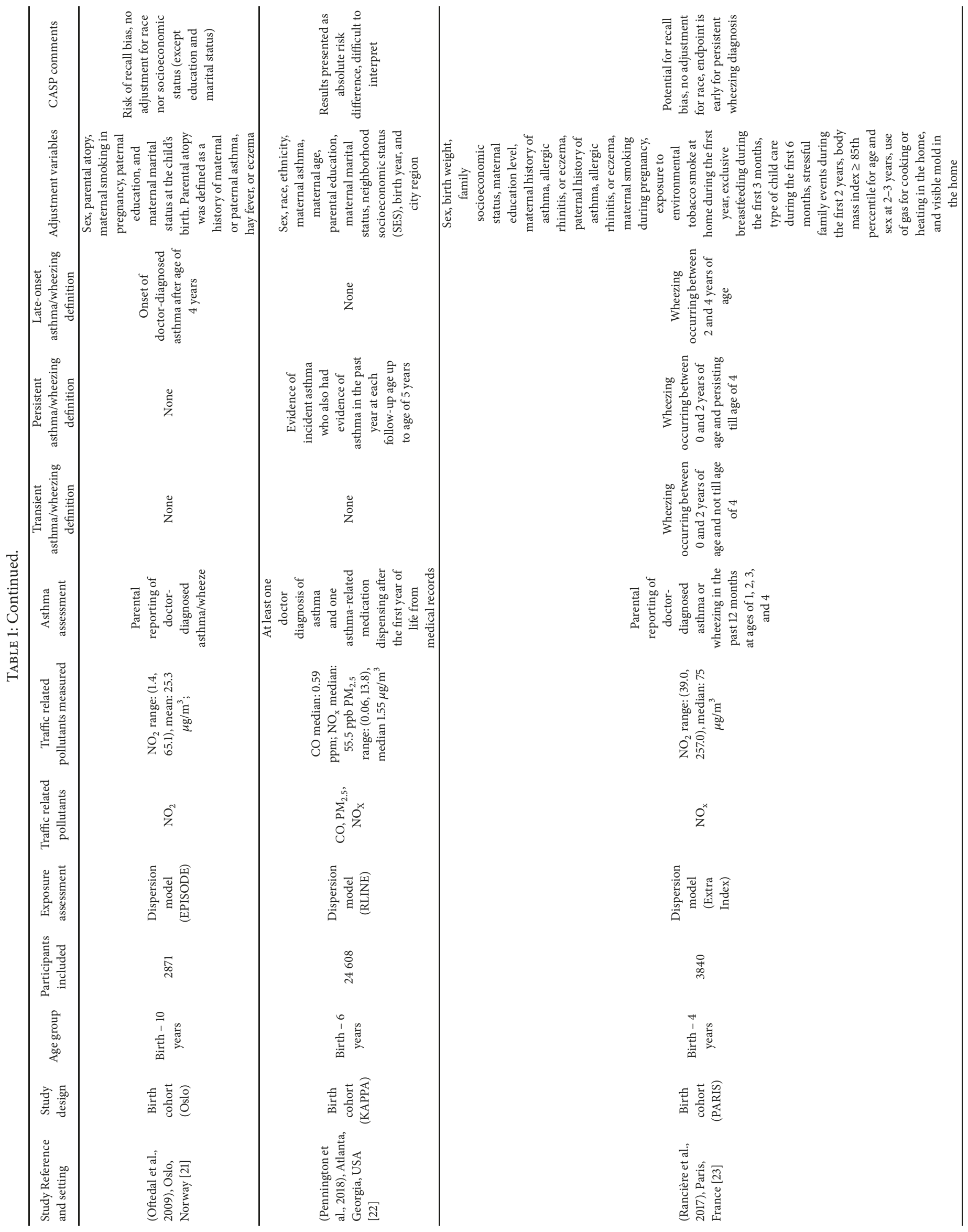




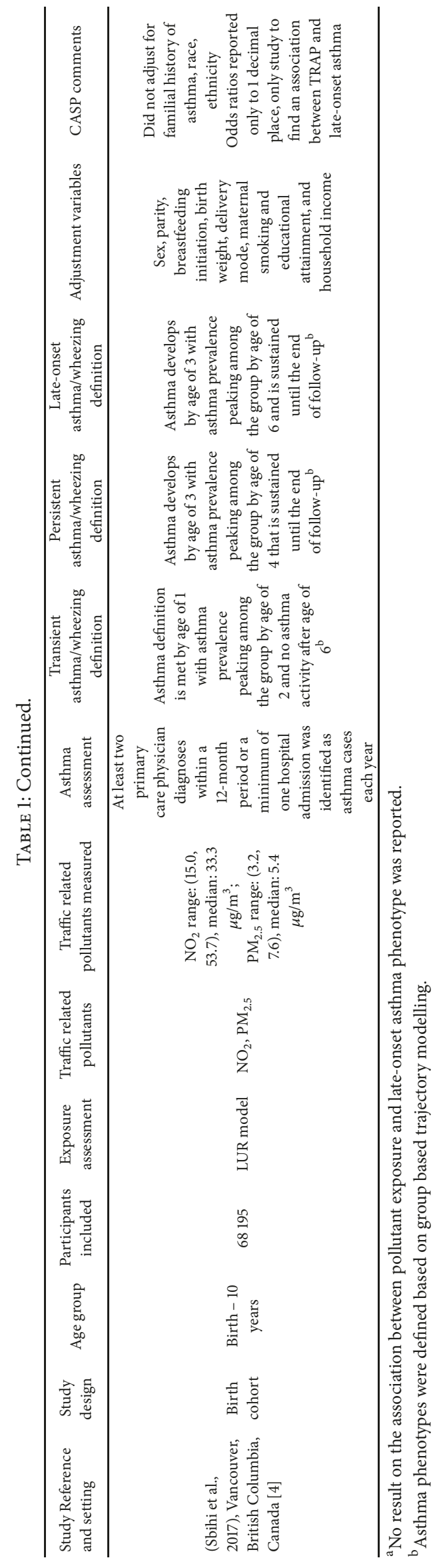




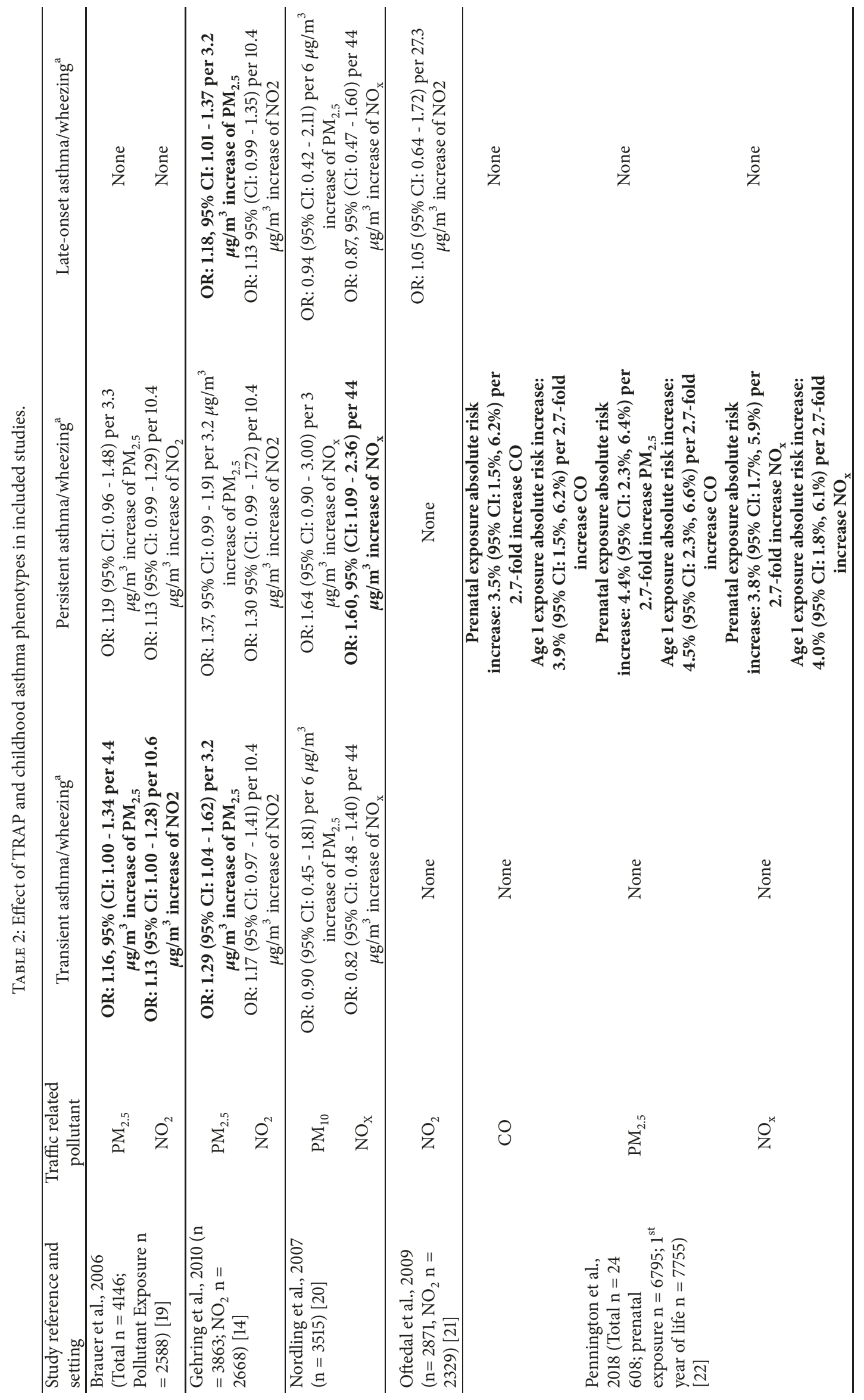




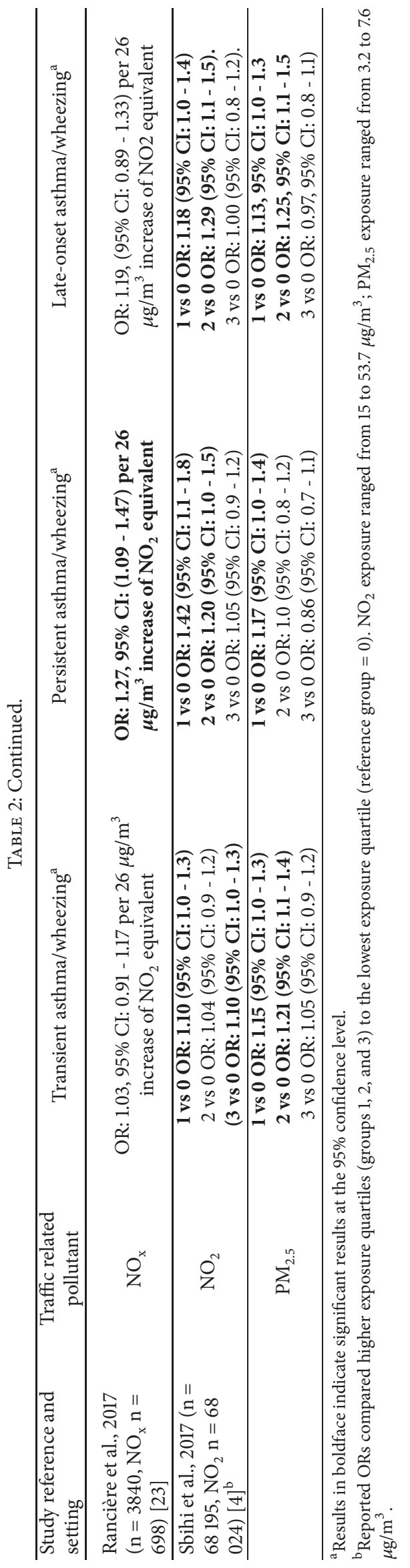


Two of three studies found no association between $\mathrm{PM}_{2.5}$ exposure and persistent wheezing or asthma phenotype [14, 19]. Sbihi et al. found that the second exposure quartile of $\mathrm{PM}_{2.5}$ was associated with persistent asthma, but there was no association between $\mathrm{PM}_{2.5}$ and the third and fourth quartiles [4].

Gehring et al. reported an association between $\mathrm{PM}_{2.5}$ and late-onset wheezing [14]. Sbihi et al. also reported that the second and third exposure quartiles were associated with lateonset asthma, although there was no association with the highest exposure quartile [4].

3.8. Effect of $P M_{10}$ on Childhood Asthma Phenotype Development. Nordling et al. reported the association between $\mathrm{PM}_{2.5}$ exposure and the development of transient wheezing, persistent wheezing, and late-onset wheezing as outcomes [20]. No significant association was found between $\mathrm{PM}_{10}$ and any of the three wheezing phenotypes.

\section{Discussion}

Although previous studies have looked at TRAP and the onset of childhood asthma, to our knowledge this is the first attempt to systematically evaluate the available literature on the effect of TRAP exposure with the development of childhood asthma or wheezing phenotypes. 7 studies were included for final analysis. The results suggest that TRAP is associated with the development of childhood transient and persistent asthma/wheezing phenotypes but may not be associated with late-onset asthma/wheezing. Nevertheless, the significance of these associations is inconsistent among the included studies and any interpretation of the results should be drawn cautiously. Stratifying studies by pollutant reduced the number of eligible studies per pollutant and made a meta-analysis unfeasible.

Early childhood exposure to TRAP has significant impacts on lung development [16]. Starting from the early postnatal period till about 1.5 years of age, bulk alveolar formation in the lungs leads to substantial structural remodeling of the lung parenchyma $[26,29]$. Microvascular maturation in the lungs also occurs starting from the early postnatal period till about 2-3 years of age. Damage to the lungs during the developmental periods has been associated with the development of long-term sequelae [27, 30, 31]. Additionally, compared to adults, children are more likely to be outdoors and active, have a higher ventilation rate, and are more likely to inhale pollutants into the distal lung, with accordingly higher exposure to TRAP [32].

$\mathrm{NO}_{2}, \mathrm{PM}_{2.5}$, and $\mathrm{PM}_{10}$ have been reported to be the primary constituents of TRAP [33], and long-term exposure to these pollutants in mice has been shown to lead to elevated levels of interleukin-6, a proinflammatory cytokine associated with inflammation and pulmonary diseases such as asthma [33-36]. TRAP exposure has also been associated with elevated expression of the Clca3 gene [33]. In animal models, expression of Clca3 has led to mucous cell metaplasia and airway hyperreactivity, leading to the development of episodic recurrent airway obstruction [37-40]. As the mucous cell metaplasia developed, it was observed that
Muc5ac was the primary airway mucin expressed, which is also characteristic of human asthma $[36,37]$. Consequently, it has been suggested that the association of TRAP with asthma onset may be due to the expression of Clca3 [33].

Genetic factors may also in part explain the heterogeneity in asthma and wheezing phenotype results presented in this review. Among children exposed to $\mathrm{NO}_{2}$, those with either a GSTP1 rs1138272 or rs1695 single nucleotide polymorphism (SNP) were found to be at an increased risk for asthma in a study combining multiple birth cohorts [41]. Additionally, high exposure to diesel exhaust particles (DEP) in children with the GST-P1 Val ${ }^{105}$ polymorphism was associated with a high risk of persistent wheezing [42]. Thus, differing genotypes among those exposed to TRAP may lead to differences in asthma or wheezing phenotypic outcomes.

Childhood asthma is a complex disease that involves many genetic and environmental factors, as well as interplay between these factors. Male children are at higher risk of childhood asthma than females, although this is reversed after puberty [43-46]. Male sex has also been shown to modify the association between prenatal $\mathrm{PM}_{2.5}$ exposure and childhood asthma onset [47]. It is uncertain whether similar interactions between sex and other forms of TRAP exist for childhood asthma onset. Exposure to other allergens such as mites is also associated with childhood asthma and can modify the risk of childhood asthma associated with TRAP [48]. Other environmental exposures such as prenatal smoke exposure, home dampness, and prenatal acetaminophen use can modify the association of genetic risk factors with childhood asthma onset [49-51].

Several limitations of this systematic review must be acknowledged. Firstly, the low number of eligible studies makes it difficult to draw any firm conclusions. Given that we assessed each pollutant separately, the number of studies per pollutant was reduced even further. Secondly, the heterogeneity in phenotype definitions and in study followup length may be a source of bias. The original phenotypic definitions from the Tucson Children's Respiratory Study measured outcomes at ages of 3 and 6 to define wheezing phenotype [5]. Given the differing follow-up length across the available studies, a child's phenotypic classification may differ between studies based on the definition used. A child with wheezing at ages of 3 and 4 but not at age of 6 would be classified as transient wheeze by Gehring et al. but would be persistent wheeze under Nordling et al., as follow-up ends at age of 4 . It is therefore important to account for these differences in phenotype definition between studies. Finally, all but two studies used parental reporting of wheezing or asthma symptoms via questionnaire response to report asthma or wheezing in children. Although standard, this may lead to recall bias in the results. These limitations suggest that further studies studying TRAP exposure and the onset of childhood asthma and wheezing phenotypes are warranted.

\section{Conclusion}

Based on the results of this systematic review, there is evidence to suggest an association between TRAP exposure and transient as well as persistent childhood asthma/wheezing 
phenotypes. Conversely, TRAP may not be associated with late-onset asthma/wheezing phenotype. However, results remain inconsistent among different studies. The low number of studies per pollutant, as well as the heterogeneity in study methods such as follow-up length and in the phenotypic definitions of asthma and wheezing used, indicates the need for further studies on this topic.

\section{Conflicts of Interest}

The authors declare that they have no conflicts of interest.

\section{Acknowledgments}

The authors would like to thank Alison Farrell who assisted with creating and performing the literature search. This work was funded by the Janeway Children's Hospital Foundation and Faculty of Medicine, Memorial University of Newfoundland.

\section{References}

[1] I. Asher and N. Pearce, "Global burden of asthma among children," The International Journal of Tuberculosis and Lung Disease, vol. 18, no. 11, pp. 1269-1278, 2014.

[2] G. A. Network, "The global asthma report 2014," Auckland, New Zealand, 2014.

[3] P. W. Sullivan, V. Ghushchyan, P. Navaratnam et al., "The national cost of asthma among school-aged children in the United States," Annals of Allergy, Asthma \& Immunology, vol. 119, no. 3, pp. 246-252.el, 2017.

[4] H. Sbihi, M. Koehoorn, L. Tamburic, and M. Brauer, "Asthma trajectories in a population-based birth cohort impacts of air pollution and greenness," American Journal of Respiratory and Critical Care Medicine, vol. 195, no. 5, pp. 607-613, 2017.

[5] F. D. Martinez, A. L. Wright, L. M. Taussig et al., "Asthma and wheezing in the first six years of life," The New England Journal of Medicine, vol. 332, no. 3, pp. 133-138, 1995.

[6] O. E. Savenije, R. Granell, D. Caudri et al., "Comparison of childhood wheezing phenotypes in 2 birth cohorts: ALSPAC and PIAMA," The Journal of Allergy and Clinical Immunology, vol. 127, no. 6, pp. 1505-e14, 2011.

[7] J. Henderson, R. Granell, J. Heron et al., "Associations of wheezing phenotypes in the first 6 years of life with atopy, lung function and airway responsiveness in mid-childhood," Thorax, vol. 63, no. 11, pp. 974-980, 2008.

[8] B. D. Spycher, M. Silverman, A. M. Brooke, C. E. Minder, and C. E. Kuehni, "Distinguishing phenotypes of childhood wheeze and cough using latent class analysis," European Respiratory Journal, vol. 31, no. 5, pp. 974-981, 2008.

[9] R. Granell, A. J. Henderson, and J. A. Sterne, "Associations of wheezing phenotypes with late asthma outcomes in the Avon Longitudinal Study of Parents and Children: A populationbased birth cohort," The Journal of Allergy and Clinical Immunology, vol. 138, no. 4, pp. 1060-1070.e11, 2016.

[10] A. H. Owora, A. B. Becker, M. Chan-Yeung et al., "Wheeze trajectories are modifiable through early-life intervention and predict asthma in adolescence," Pediatric Allergy and Immunology, vol. 29, no. 6, pp. 612-621, 2018.
[11] M. B. Reddy and R. A. Covar, "Asthma phenotypes in childhood," Current Opinion in Allergy and Clinical Immunology, vol. 16, no. 2, pp. 127-134, 2016.

[12] M. Brauer, G. Hoek, P. Van Vliet et al., "Air pollution from traffic and the development of respiratory infections and asthmatic and allergic symptoms in children," American Journal of Respiratory and Critical Care Medicine, vol. 166, no. 8, pp. 1092-1098, 2002.

[13] M. Jerrett, K. Shankardass, K. Berhane et al., "Traffic-related air pollution and asthma onset in children: a prospective cohort study with individual exposure measurement," Environmental Health Perspectives, vol. 116, no. 10, pp. 1433-1438, 2008.

[14] U. Gehring, A. H. Wijga, M. Brauer et al., "Traffic-related air pollution and the development of asthma and allergies during the first 8 years of life," American Journal of Respiratory and Critical Care Medicine, vol. 181, no. 6, pp. 596-603, 2010.

[15] J. J. Kim, S. Smorodinsky, M. Lipsett, B. C. Singer, A. T. Hodgson, and B. Ostro, "Traffic-related air pollution near busy roads: The East Bay Children's Respiratory Health Study," American Journal of Respiratory and Critical Care Medicine, vol. 170, no. 5, pp. 520-526, 2004.

[16] N. A. Clark, P. A. Demers, C. J. Karr et al., "Effect of early life exposure to air pollution on development of childhood asthma," Environmental Health Perspectives, vol. 118, no. 2, pp. 284-290, 2010.

[17] H. Khreis, C. Kelly, J. Tate, R. Parslow, K. Lucas, and M. Nieuwenhuijsen, "Exposure to traffic-related air pollution and risk of development of childhood asthma: A systematic review and meta-analysis," Environment International, 2016.

[18] G. Bowatte, C. Lodge, A. J. Lowe et al., "The influence of childhood traffic-related air pollution exposure on asthma, allergy and sensitization: A systematic review and a metaanalysis of birth cohort studies," Allergy: European Journal of Allergy and Clinical Immunology, vol. 70, no. 3, pp. 245-256, 2015.

[19] M. Brauer, G. Hoek, H. A. Smit et al., "Air pollution and development of asthma, allergy and infections in a birth cohort," European Respiratory Journal, vol. 29, no. 5, pp. 879-888, 2007.

[20] E. Nordling, N. Berglind, E. Melén et al., "Traffic-related air pollution and childhood respiratory symptoms, function and allergies," Epidemiology, vol. 19, no. 3, pp. 401-408, 2008.

[21] B. Oftedal, W. Nystad, B. Brunekreef, and P. Nafstad, "Longterm traffic-related exposures and asthma onset in schoolchildren in Oslo, Norway," Environmental Health Perspectives, vol. 117, no. 5, pp. 839-844, 2009.

[22] A. F. Pennington, M. J. Strickland, M. Klein et al., "Exposure to Mobile Source Air Pollution in Early-life and Childhood Asthma Incidence: The Kaiser Air Pollution and Pediatric Asthma Study," Epidemiology, pp. 22-30, 2018.

[23] F. Rancière, N. Bougas, M. Viola, and I. Momas, "Early exposure to traffic-related air pollution, respiratory symptoms at 4 years of age, and potential effect modification by parental allergy, stressful family events, and sex: A prospective follow-up study of the Paris birth cohort," Environmental Health Perspectives, vol. 125, no. 4, pp. 737-745, 2017.

[24] D. Moher, A. Liberati, J. Tetzlaff, and D. G. Altman, "Preferred reporting items for systematic reviews and meta-analyses: the PRISMA statement," Annals of internal medicine, vol. 151, no. 4, pp. 264-269, 2009. 
[25] E. S. Schultz, A. A. Litonjua, and E. Melén, "Effects of LongTerm Exposure to Traffic-Related Air Pollution on Lung Function in Children," Current Allergy and Asthma Reports, vol. 17, no. 6, 2017.

[26] T. B. Zeltner and P. H. Burri, "The postnatal development and growth of the human lung. II. Morphology," Respiration Physiology, vol. 67, no. 3, pp. 269-282, 1987.

[27] N. Dik, R. B. Tate, J. Manfreda, and N. R. Anthonisen, "Risk of physician-diagnosed asthma in the first 6 years of life," CHEST, vol. 126, no. 4, pp. 1147-1153, 2004.

[28] Critical Appraisal Skills Programme, CASP checklists. Critical Appraisal Skills Programme (CASP): Making sense of evidence, 2014, https://casp-uk.net/wp-content/uploads/2018/01/ CASP-Cohort-Study-Checklist.pdf.

[29] T. B. Zeltner, J. H. Caduff, P. Gehr, J. Pfenninger, and P. H. Burri, "The postnatal development and growth of the human lung. I. Morphometry," Respiration Physiology, vol. 67, no. 3, pp. 247267, 1987.

[30] M. Brauer, C. Lencar, L. Tamburic, M. Koehoorn, P. Demers, and C. Karr, "A cohort study of traffic-related air pollution impacts on birth outcomes," Environmental Health Perspectives, vol. 116, no. 5, pp. 680-686, 2008.

[31] E. L. Avol, W. James Gauderman, S. M. Tan, S. J. London, and J. M. Peters, "Respiratory effects of relocating to areas of differing air pollution levels," American Journal of Respiratory and Critical Care Medicine, vol. 164, no. 11, pp. 2067-2072, 2001.

[32] M. Brauer, C. Reynolds, and P. Hystad, "Traffic-related air pollution and health in Canada," Canadian Medical Association Journal, vol. 185, no. 18, pp. 1557-1558, 2013.

[33] J. Yang, Y. Chen, Z. Yu, H. Ding, and Z. Ma, "Changes in gene expression in lungs of mice exposed to traffic-related air pollution," Molecular and Cellular Probes, vol. 39, pp. 33-40, 2018.

[34] A. Hajat, M. Allison, A. V. Diez-Roux et al., "Long-term exposure to air pollution and markers of inflammation, coagulation, and endothelial activation a repeat-measures analysis in the multi-ethnic study of atherosclerosis (MESA)," Epidemiology, vol. 26, no. 3, pp. 310-320, 2015.

[35] B. Hoffmann, S. Moebus, N. Dragano et al., "Chronic residential exposure to particulate matter air pollution and systemic inflammatory markers," Environmental Health Perspectives, vol. 117, no. 8, pp. 1302-1308, 2009.

[36] M. Rincon and C. G. Irvin, "Role of IL-6 in asthma and other inflammatory pulmonary diseases," International Journal of Biological Sciences, vol. 8, no. 9, pp. 1281-1290, 2012.

[37] A. C. Patel, T. J. Brett, and M. J. Holtzman, "The role of CLCA proteins in inflammatory airway disease," Annual Review of Physiology, vol. 71, pp. 425-449, 2009.

[38] F. C. Lowell, "Observations on heaves. An asthma-like syndrome in the horse," Journal of Allergy, vol. 35, no. 4, pp. 322330, 1964.

[39] E. Davis and B. R. Rush, "Equine recurrent airway obstruction: pathogenesis, diagnosis, and patient management," Veterinary Clinics of North America - Equine Practice, vol. 18, no. 3, pp. 453$467,2002$.

[40] R. Léguillette, "Recurrent airway obstruction - Heaves," Veterinary Clinics of North America - Equine Practice, vol. 19, no. 1, pp. 63-86, 2003.

[41] E. A. MacIntyre, M. Brauer, E. Melén et al., "GSTP1 and TNF gene variants and associations between air pollution and incident childhood asthma: The traffic, asthma and genetics
(TAG) study," Environmental Health Perspectives, vol. 122, no. 4, pp. 418-424, 2014.

[42] K. T. Schroer, J. M. Biagini Myers, P. H. Ryan et al., "Associations between Multiple Environmental Exposures and Glutathione S-transferase P1 on Persistent Wheezing in a Birth Cohort," Journal of Pediatrics, vol. 154, no. 3, pp. 401-e1, 2009.

[43] S. S. Szentpetery, O. Gruzieva, E. Forno et al., "Combined effects of multiple risk factors on asthma in school-aged children," Respiratory Medicine, vol. 133, pp. 16-21, 2017.

[44] L. Fu, R. J. Freishtat, H. Gordish-Dressman et al., "Natural progression of childhood asthma symptoms and strong influence of sex and puberty," Annals of the American Thoracic Society, vol. 11, no. 6, pp. 898-907, 2014.

[45] A. F. Pennington, M. J. Strickland, M. Klein, C. Drews-Botsch, C. Hansen, and L. A. Darrow, "Caesarean delivery, childhood asthma, and effect modification by sex: An observational study and meta-analysis," Paediatric and perinatal epidemiology, 2018.

[46] R. Arathimos, R. Granell, J. Henderson, C. L. Relton, and K. Tilling, "Sex discordance in asthma and wheeze prevalence in two longitudinal cohorts," PLoS ONE, vol. 12, no. 4, 2017.

[47] H.-H. L. Hsu, Y.-H. M. Chiu, B. A. Coull et al., "Prenatal particulate air pollution and asthma onset in urban children: Identifying sensitive windows and sex differences," American Journal of Respiratory and Critical Care Medicine, vol. 192, no. 9, pp. 1052-1059, 2015.

[48] I. J. Wang, T. H. Tung, C. S. Tang, and Z. H. Zhao, "Allergens, air pollutants, and childhood allergic diseases," International journal of hygiene and environmental health, vol. 219, no. 1, pp. 66-71, 2016.

[49] A. Sadeghnejad, W. Karmaus, S. H. Arshad, R. Kurukulaaratchy, M. Huebner, and S. Ewart, "IL13 gene polymorphisms modify the effect of exposure to tobacco smoke on persistent wheeze and asthma in childhood, a longitudinal study," Respiratory Research, vol. 9, 2008.

[50] S. O. Shaheen, R. B. Newson, S. M. Ring, M. J. Rose-Zerilli, J. W. Holloway, and A. J. Henderson, "Prenatal and infant acetaminophen exposure, antioxidant gene polymorphisms, and childhood asthma," The Journal of Allergy and Clinical Immunology, vol. 126, no. 6, pp. 1141.e7-1148.e7, 2010.

[51] C. H. Tsai, K. Y. Tung, C. H. Chen, and Y. L. Lee, “Tumour necrosis factor G-308A polymorphism modifies the effect of home dampness on childhood asthma," in Occupational and Environmental Medicine, 2011. 


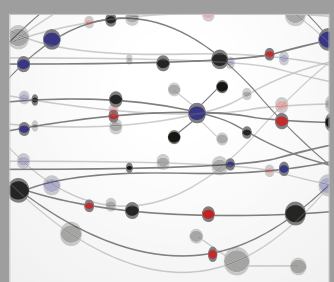

The Scientific World Journal
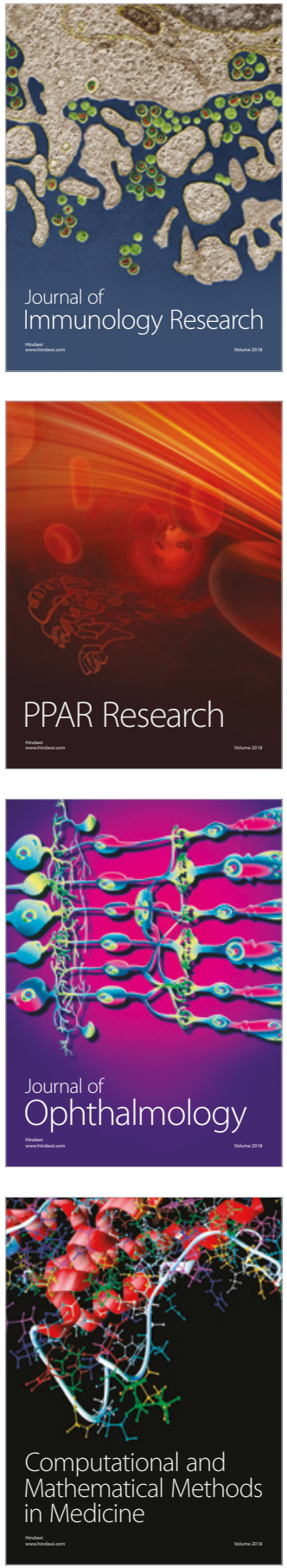

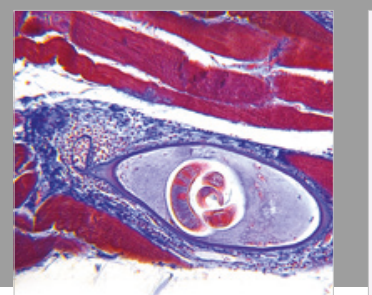

Gastroenterology Research and Practice

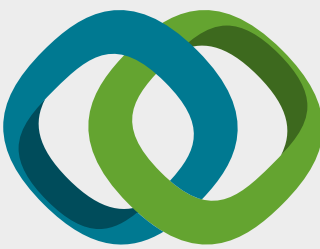

\section{Hindawi}

Submit your manuscripts at

www.hindawi.com
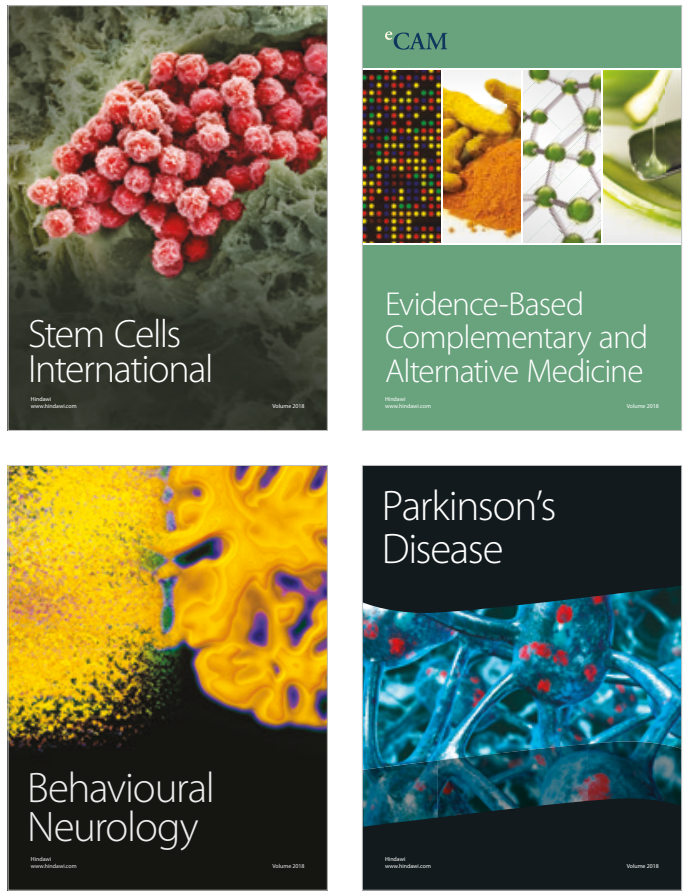

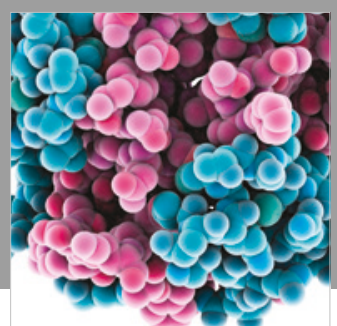

ournal of

Diabetes Research

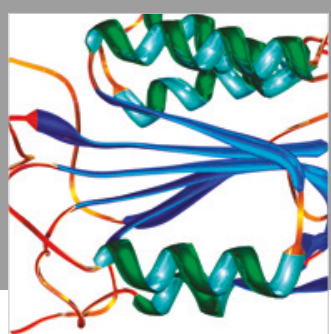

Disease Markers
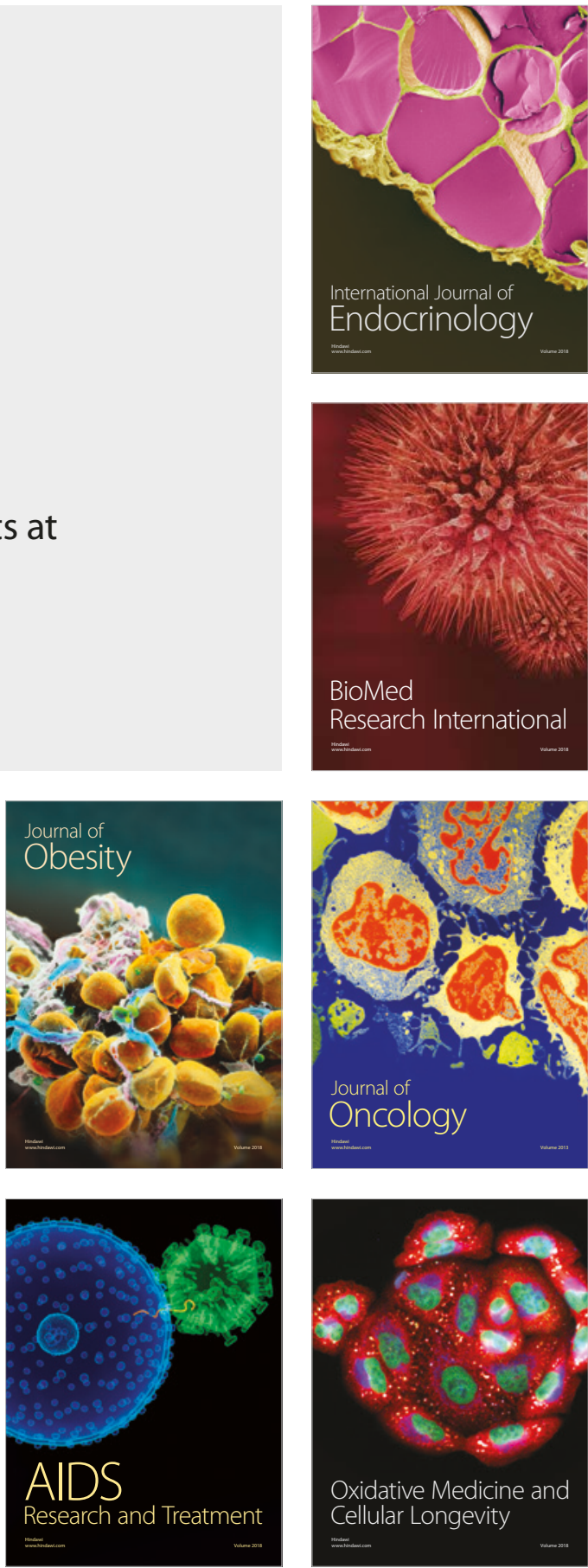